\title{
Actitud e intención emprendedora en estudiantes de administración de empresas y de contaduría pública*
}

\author{
Édgar Julián Gálvez Albarracín ${ }^{* *}$ \\ Rafael Andrés Guauña Aguilar ${ }^{\star *}$ \\ Rafael Ravina Ripoll ${ }^{\star \star * *}$
}

Fecha de recibido: 13 de septiembre de 2018 Fecha de aprobado: 5 de marzo de 2019

Para citar: Gálvez Albarracín, E. J., Guauña Aguilar, R. A., \& Ravina Ripoll, R. (2020). Actitud e intención emprendedora en estudiantes de administración de empresas y de contaduría pública. Universidad \& Empresa, 22(38), 79-105. https://doi.org/10.12804/ revistas.urosario.edu.co/empresa/a.7230

* Este artículo de investigación científica se deriva del trabajo de grado presentado en el año 2016 por Rafael GuauñaAguilar bajo la dirección de Édgar Gálvez-Albarracín, en el marco de la Maestría en Administración de Empresas de la Universidad del Valle en Cali, Colombia.

** Doctor en Administración y Dirección de Empresas, Universidad Politécnica de Cartagena, España. Magíster en Creación de Empresas y Gestión de Proyectos Innovadores, Universidad Montesquieu Bordeaux IV, Francia. Miembro del Grupo de Investigación en Humanismo y Gestión y profesor titular, Facultad de Ciencias de la Administración, Universidad del Valle, Cali, Colombia. Correo electrónico: edgar.galvez@correounivalle.edu.co ORCID: https://orcid.org/0000-0003-3972-2310_

*** Magíster en Administración de Empresas, Universidad del Valle. Coordinador de Fortalecimiento Empresarial, Cámara de Comercio del Cauca. Profesor hora catedra, Facultad de Ciencias de la Administración, Universidad del Valle, Sede Norte del Cauca, Colombia. Correo electrónico: formacionstder@cccauca.org.co

**** Doctor en Historia, Universidad de Cádiz, España. Magíster en Economía Social y Desarrollo Local. Profesor asociado, Organización de Empresa de la Universidad de Cádiz, España. Correo electrónico: rafael.ravina@uca.es. ORCID: http:// orcid.org/0000-0001-7007-3123. 


\title{
Resumen
}

Múltiples estudios demuestran que diferentes variables influyen en la disposición de los individuos para realizar alguna acción, en ese sentido, y tomando como base la Teoría del Comportamiento Planificado (TCP), este trabajo busca describir el nivel de intención de crear empresas propias de los estudiantes universitarios, y cómo este es afectado por su percepción sobre los estímulos y barreras implicadas en ello. Para esto, se tomó una muestra de 246 alumnos de administración de empresas y de contaduría pública de la Sede Norte del Cauca de la Universidad del Valle en Colombia, zona geográfica que históricamente ha presentado problemas económicos y sociales. Los resultados descriptivos muestran que alrededor de un 70\% de los alumnos estaban interesados en ser empresarios, siendo su principal motivación para emprender obtener seguridad familiar y personal, pero lo que más los desmotiva es la falta de capital para ello; por su parte, las diferencias de medias y regresiones lineales aplicadas como técnicas estadísticas confirman que a mayor motivación global mayor intención emprendedora en las personas. Se concluye que, debido especialmente a las recompensas que perciben, para los futuros profesionales de ciudades pequeñas la actividad empresarial se constituye también en una importante alternativa de desarrollo.

Palabras clave: emprendimiento universitario, creación de empresas, Teoría del Comportamiento Planificado, motivaciones, barreras percibidas.

\section{Attitude and Entrepreneurial Intention in Business Administration and Public Accounting Students}

\begin{abstract}
Multiple studies show that diverse variables influence the disposition of subjects to perform some action, in that sense and based on the theory of planned behavior (TCP), this work seeks to describe the level of intention to create companies owned by university students and how this is affected by their perception of the stimuli and barriers involved in it. In this sense, a sample of 246 students of business administration and public accounting was taken from the North Headquarters of Cauca of the Universidad del Valle in Colombia, a geographical area that has historically presented economic and social problems. The descriptive results show that around $70 \%$ of the students were interested in being entrepreneurs, being their main motivation to undertake family and personal security and what most discourages the lack of capital for it; meanwhile, the differences in means and linear regressions applied as statistical techniques confirm that the greater the global motivation, the greater the entrepreneurial intention in people. The conclusion of the article is that, due to the rewards they receive, for small cities' future professionals, business activity is also an essential development alternative.
\end{abstract}

Keywords: University entrepreneurship, business creation, Theory of Planned Behavior, motivations, perceived barriers.

\section{Atitude e intenção empreendedora em estudantes de Administração de Empresas e de Contadoria pública}

\section{Resumo}

Múltiplos estudos demostram que diferentes variáveis influem na disposição dos indivíduos para realizar alguma ação, nesse sentido e tomando como base a Teoria do Comportamento Planificado (TCP) este trabalho busca descrever o nível de intenção de criar empresas próprias dos estudantes universitários, e como este é afetado por sua percepção sobre os estímulos e barreiras implicadas em isso. Em dito sentido tomara-se uma amostra de 246 estudantes de Administração de Empresas e de Contadoria Pública da Sede Norte de Cauca da Universidad del Valle na Colômbia, zona geográfica que historicamente tem apresentado problemas econômicos e sociais. Os resultados descritivos mostram que ao redor de um 70\% dos estudantes estavam interessados em ser empresários, 
sendo sua principal motivação para empreender, obter segurança familiar e pessoal e o que mais os desmotiva é a falta de capital para isto; por sua parte, as diferenças de médias e regressões lineares aplicadas como técnicas estatísticas confirmam que à maior motivação global maior intenção empreendedora nas pessoas. Se conclui que devido especialmente às recompensas que percebem, para os futuros profissionais de cidades pequenas a atividade empresarial se constitui também em uma importante alternativa de desenvolvimento.

Palavras-chave: empreendimento universitário; criação de empresas; Teoria do Comportamento Planificado; motivações; barreiras percebidas.

\section{Introducción}

El emprendimiento y su manifestación en la creación de empresas son considerados dinamizadores del desarrollo de las naciones y las regiones, por lo que el sector público, el privado y la academia muestran interés creciente por entender dichos fenómenos y los elementos que los estimulan o desfavorecen (Díaz, Fernández, Almodóvar, Díaz y Fernández, 2017; Varela, 2008; Audretsch y Belitski, 2007), buscando que cada vez más estudiantes y profesionales universitarios se conviertan en empresarios (Shirokova, Tsukanova y Morris, 2018; García, Mendoza y Romo, 2017; Goyanes, 2015; Joensuu, Varamäki y Viljamaa, 2015; Bae, Qian, Miao y Fiet, 2014; Coque, Díaz y López, 2013; González, 2004).

En dicho contexto, es importante tener en cuenta que en cualquier proceso de emprendimiento intervienen al menos dos factores: las personas y el entorno general y empresarial, y que para autores como Vainrub (2009) y Timmons y Spinelli (2004) el primero es el eje central, por lo que su potencial emprendedor es estudiado desde enfoques como sus aptitudes (Mora, 2011) y sus perfiles sociales y psicosociales (Novillo, Sarmiento, Ollague y Ramón, 2017; Moriano, Trejo y Palací, 2001).

De acuerdo con el modelo conceptual del Global Entrepreneurship Monitor (GEM), estudio que mide la tasa de actividad emprendedora (TEA) en diferentes países y regiones, una de las fases en la creación de empresas es la de intención, en la cual a los individuos que están considerando hacerlo se les define como 'empresarios intencionales'. En ese sentido, numerosas investigaciones como las de Laguía, Moriano, Molero y Gámez (2017), Valencia, Cadavid, Ríos y Awad (2012), Rueda, Fernández y Herrero (2012), y Sánchez, Lanero y Yurrebaso (2005) han confirmado que la intención es una buena predictora del inicio de empresas. 
No obstante, el informe GEM 2016 muestra para Colombia que, mientras un 53\% las personas encuestadas en este país eran empresarios intencionales, solo el 16.3\% habían iniciado efectivamente actividades empresariales (Buelvas, Franco, García, Gómez, López, Matíz, ...Varela, 2017), habiéndose desaprovechado o perdido en consecuencia el restante $26.7 \%$ de las intenciones de crear nuevas empresas, situación que debe interesar y preocupar a su clase dirigente e intelectual.

De esta manera, a nivel mundial, numerosos trabajos como los de Gorgievski, Stephan, Laguna y Moriano (2018), Chantson y Urban (2018), Galvão, Marques y Marques (2018), Valencia, Montoya y Montoya (2015), Liñán y Chen (2009), Zhao, Seibert y Hills (2005) y Krueger, Reilly y Carsrud (2000) han confirmado la utilidad de los conceptos aportados por la Teoría del Comportamiento Planificado desarrollada por Ajzen en 1991, para identificar y describir los factores que estimulan o desalientan a los individuos de diferentes condiciones y características la intención empresarial o de crear empresas, a lo que en este documento se le denominará como 'intención emprendedora'; sin embargo, dichos estudios se han aplicado principalmente en grandes ciudades, sin profundizar en las más pequeñas, que generalmente difieren en su ecosistema empresarial respecto a las primeras.

En ese escenario se encuentra la Universidad del Valle, Sede Norte del Cauca, ubicada en Colombia en el municipio de Santander de Quilichao. La institución atiende a su población y a la de otros 13 municipios de igual densidad (menos de 100000 habitantes) en los Departamentos del Cauca y en el sur del Valle del Cauca, los cuales son importantes para la región y el país por su potencial económico, debido a sus importantes recursos naturales, pero también por su problemática social relacionada con temas de inseguridad y desempleo, entre otros factores.

De acuerdo con lo señalado, este trabajo de investigación busca contribuir a llenar el vacío de literatura descrito en relación con el emprendimiento universitario en ciudades no capitales, respondiendo a la pregunta ¿cuál es el nivel de intención emprendedora en los estudiantes de contaduría pública y administración de empresas de la Universidad del Valle, Sede Norte del Cauca, y cómo su actitud frente al tema influye positiva o negativamente en ello?

El artículo está estructurado de tal manera que en la primera parte se presenta el marco teórico correspondiente, los estudios previos encontrados y las hipótesis de investigación; 
en la segunda se describe la metodología utilizada; en la tercera se muestran y discuten los resultados del trabajo; y por último se plantean sus conclusiones, limitaciones y futuras líneas de trabajo.

\section{Marco teórico, estudios previos e hipótesis de la investigación}

A continuación, se describen los principales aspectos teórico-conceptuales que articulan la presente investigación.

\subsection{Emprendimiento, emprendedor e intención emprendedora}

El emprendimiento y los emprendedores son objeto de estudio debido a su relevante papel en el cambio y el desarrollo socioeconómico (Diez, 2016; Park y Duarte, 2015), es así que Schumpeter (1950) los vincula con el concepto de 'empresario innovador', entendiéndolo como aquel que es capaz de romper la inercia de los mercados para renovarlos de manera permanente.

El termino 'emprendimiento', que busca retomar el que en francés se denomina 'entrepreneuriat' y en inglés 'entrepreneurship', es definido en el Diccionario de la Real Academia de la Lengua Española (DRAE) en una de sus acepciones como "acción de emprender", y a la acción de 'emprender' como "acometer y comenzar una obra, un negocio, un empeño, especialmente si encierran dificultad o peligro", y al 'emprendedor' como quien "emprende con resolución acciones o empresas innovadoras" (RAE, 2017).

Diferentes autores se refieren también al emprendimiento con otros términos como 'actividad emprendedora' o 'espíritu empresarial' y señalan que tienen tres elementos fundamentales: 1) el individuo o grupo de individuos que lo ejercen, 2) el medio o entorno en el cual se desarrolla, y 3) la acción o actividad emprendedora como tal. Por ello, para entenderlo como fenómeno económico y social ha sido estudiado desde diferentes enfoques o corrientes, entre ellas la económica, la socio-psicológica y la comportamental (Gómez, 2010). 
En relación con la creación de empresas, Shapero (1984) y Alonso (2012) señalaban que esta podía estar animada o desestimulada por las percepciones de deseabilidad y de factibilidad que el emprendedor tuviera de dicha acción. Posteriormente, autores como Krueger (2000) y Liñán (2004) señalaron la importancia de, además de los rasgos de personalidad y los factores demográficos, agregar a dicho análisis elementos como la intención, lo cual podría hacerse mediante teorías como la del comportamiento planificado de Ajzen (1991).

\subsection{Teoría del Comportamiento Planificado (TCP)}

La Teoría del Comportamiento Planificado (TCP), utilizada en múltiples investigaciones para medir la intención emprendedora de las personas (Valencia, Montoya y Montoya, 2016), fue propuesta por el psicólogo social Icek Ajzen en 1991 como complemento de la teoría de la acción razonada (Ajzen y Fishben, 1980), buscando perfeccionar el marco de comprensión de los antecedentes de las acciones de los individuos. La TCP, que ha derivado en diversos cuestionarios como los utilizados por Liñán, Moriano y Jaén (2016), postula como predictores de la creación de empresas los siguientes factores: la actitud hacia el comportamiento, la norma subjetiva y el control percibido o autoeficacia. La figura 1 muestra el modelo conceptual descrito, allí se puede observar cómo los tres factores señalados se influencian mutuamente y a su vez generan la intención, y esta el comportamiento.

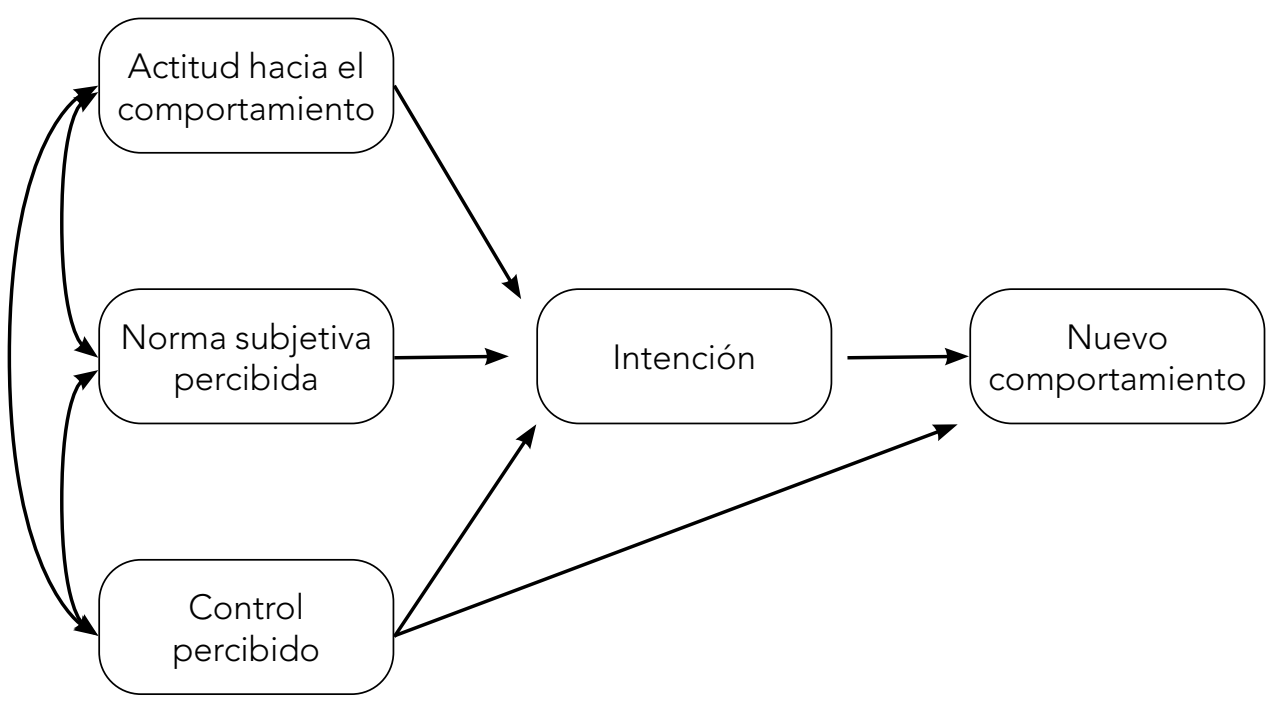

Figura 1. Teoría del Comportamiento Planificado (TCP)

Fuente: elaboración propia con base en Ajzen (1991). 
Ajzen (1991) define estos términos. La 'actitud' hacia el comportamiento es definida como aquellos factores que impulsan o desalientan la realización de este, es decir los motivos y las barreras percibidas por las personas para realizar una acción determinada.

La 'norma subjetiva' la entiende como la percepción que tienen los individuos respecto a la valoración que la sociedad hace del comportamiento objeto de análisis, es decir, si es observada como algo positivo y destacable y por ello se convierte en un aliciente para que se desarrolle, o por el contrario se rechaza y condena por lo que se ve desestimulada.

Por último, el 'control percibido' o autoeficacia lo define como la percepción o grado de confianza que tiene el individuo en relación con su capacidad y preparación para realizar una determinada actividad.

En este artículo se revisará específicamente el primer factor de la teoría de Ajzen (1991), sumando esfuerzos así a la línea de los trabajos de Diez (2016), Pallí y Martínez (2004) y Kouriloff (2000), buscándose en este caso conocer el nivel de los motivos y barreras percibidas por los sujetos observados, es decir, su actitud en relación con la creación de empresas y el impacto que ella tiene en su intención emprendedora.

A continuación, se relacionan trabajos académicos encontrados en relación con la TcP, la actitud y la intención emprendedora, que aportan elementos conceptuales o empíricos a la presente investigación:

\subsection{Estudios previos sobre la TCP, la actitud y la intención emprendedora}

Sánchez, Lanero y Yurrebaso (2005), para desarrollar un modelo psicológico integrador sobre la formación de intenciones emprendedoras, analizan sus variables determinantes en la Universidad de Salamanca, España, hallando que, de los factores frecuentemente estudiados, los más importantes fueron la autoeficacia, la propensión al riesgo y la proactividad. Por su parte, Valencia, Cadavid, Ríos y Awad (2010), en un trabajo con 477 estudiantes de la Facultad de Minas de la Universidad Nacional de Colombia, Sede Medellín (Fmunalmed), en la búsqueda de desarrollar un programa de emprendimiento que estimule efectivamente la creación de empresas, confirman la necesidad de conocer tanto sus motivaciones como sus barreras percibidas. 
Dewi y Martdianty (2012) pusieron a prueba la TCP para explicar la intención de iniciar empresa en seis universidades de Indonesia, con una muestra de 600 estudiantes, sus resultados mostraron que dicha teoría fue útil para predecir la intención emprendedora simultáneamente en las seis universidades y en el total de los alumnos observados. A su vez, la investigación de Rueda, Fernández y Herrero (2012) con 525 estudiantes de la Facultad de Ciencias Económicas y Empresariales de la Universidad de Cantabria, España, utilizó la misma teoría para describir cómo los elementos de la TCP influyen en la intención emprendedora de sus estudiantes, los resultados muestran que está relacionada con las habilidades y capacidades del individuo para desarrollar un proyecto empresarial, la influencia social percibida en su entorno próximo, así como con sus creencias.

Yang (2013), con una muestra de 1330 estudiantes chinos de la Universidad de Finanzas y Economía Jiangxi, encontró que la actitud es el predictor más eficaz de la intención emprendedora, seguido de la norma subjetiva y la autoeficacia.

Por su parte, Rueda, Sánchez, Blanco y Fernández (2013) publican los resultados de una investigación realizada en España, en la cual, al utilizar la тсP, buscan conocer los factores que motivan o frenan la intención emprendedora de los estudiantes universitarios, discriminándolos según el género de los individuos. Entre sus resultados, encuentran que, en cuanto a las motivaciones, las mujeres le dan más importancia que los hombres a las recompensas personales (crecimiento personal, reconocimiento público, probar que se es capaz de hacerlo) y menos que ellos a las recompensas económicas. Por otra parte, se encontró que ellas ven como una barrera más importante el temor a fracasar, y no es tan relevante el tener pocas horas libres por trabajar mucho tiempo en las nuevas empresas.

Por su lado, Chan, Escalante y Robles (2015) publican un estudio en el que comparan la intención emprendedora en estudiantes de carreras económicas y administrativas en dos universidades ubicadas respectivamente en México y Puerto Rico. Sus hallazgos muestran que los factores que más favorecen el querer crear empresa son el buen manejo de problemas, la creatividad, la seguridad y la aceptación del riesgo, siendo este último factor el que marca la diferencia entre los alumnos de los dos países.

Vargas y Bedoya (2015) publican su trabajo de grado de Maestría en Administración de Empresas en el que por medio de la TCP analizaron los factores psicológicos que influ- 
yen en la intención emprendedora de 292 estudiantes de nivel tecnológico del Servicio Nacional de Aprendizaje de Colombia (SENA). Sus resultados muestran que el $71 \%$ de los estudiantes manifestaron que era probable o totalmente probable que llegaran a crear empresa, siendo dicha intención más alta en los estudiantes de contabilidad y finanzas que en los de procesos administrativos.

En este estudio, la media para las motivaciones analizadas fue de 4.35, en una escala de 1 a 5 . El factor individual más alto fue la "seguridad familiar y personal" con $4.53 \mathrm{y}$ el más bajo las "recompensas personales" con 4.05; así mismo, la media de las barreras percibidas fue de 3.4, siendo la "falta de capital" la más alta con 4.08 y la más baja la de quedar "con tiempo limitado para otras actividades" con 3.03.

En cuanto al efecto de la actitud sobre la intención emprendedora, encontraron que las motivaciones la influyen positivamente y que el factor que más lo hace es "invertir y conseguir un patrimonio personal" seguido de "hacer realidad alguno de mis sueños"; de igual manera, las barreras la desestimulan son el "temor a fracasar o quedar en ridículo" y "el riesgo económico", en mayor medida y, en menor rango, "la falta de capacitación, asesoría y acompañamiento”.

Marín, Rubio y Sánchez (2015) publican los resultados de un estudio realizado con 335 alumnos de la Universidad de Murcia, España, en el que analizan los determinantes de la intención emprendedora de los estudiantes universitarios. Entre sus aportes puede citarse que logran demostrar la importancia que sobre la intención emprendedora tiene la actitud y concluyen que, si las universidades aumentan el número de estudiantes con una actitud positiva hacia la creación de empresas, lograrán a futuro tener muchos más que consideren la vida empresarial como una valiosa opción de desarrollo profesional.

Guauña (2015) publica los resultados de su trabajo de grado de Maestría en el cual, con una muestra de 246 estudiantes de la Facultad de Ciencias de la Administración de la Universidad del Valle en Colombia, analiza los factores psicológicos que influyen en la intención emprendedora. Los resultados muestran que las motivaciones tienen impacto positivo en el deseo de crear empresas por parte de los alumnos universitarios. 
Fernández (2017) publica los resultados de su trabajo de grado para optar por su título en la Facultad de Turismo y Finanzas de la Universidad de Sevilla, España, en la cual mediante la ТСР y con una muestra de 204 alumnos pretendía encontrar las diferencias entre la intención emprendedora entre hombres y mujeres. Los resultados muestran que los alumnos del género masculino tienen mayor intención que las del femenino, lo cual intentan explicar en la percepción que las mismas mujeres tienen de las ocupaciones que deben asumir en la sociedad como ser amas de casa y en el tener mayor dificultad para acceder a financiamiento bancario; de igual manera, encuentran que los estudiantes que no tienen algún tipo de experiencia laboral tienen menos intención que los que sí.

Por su parte, Jaimes, Jaramillo y Pérez (2017) publican los resultados de una investigación en la cual buscan identificar los factores que inciden en la intención emprendedora de los estudiantes de las áreas económico-administrativas del Centro Universitario Temascaltepec de la Universidad Autónoma del Estado de México, para ello toman una muestra de 342 estudiantes de las licenciaturas en Administración, Contaduría e Informática Administrativa. Los resultados revelan que los factores sociodemográficos que inciden en la intención emprendedora de los estudiantes son su semestre, estado civil, edad y conocimiento del programa de emprendimiento, y los factores psicológicos más influyentes son su propensión al riesgo, autoestima e innovación.

Díaz, Fernández, Almodóvar, Díaz y Fernández (2017) publican los resultados del informe Guess, donde, entre otros factores y también mediante la TCP, miden en una muestra 2497 estudiantes la intención emprendedora en la Universidad de Extremadura en España. Encuentran que, en una escala de 1 a 7 donde 1 es nada dispuestos a crear empresa y 7 totalmente, la media del total de encuestados estuvo alrededor de 4 , lo que está por encima del valor medio (3.5). Sin embargo, también hallan que solo el $4.7 \%$ de los estudiantes quisieran implicarse en crear empresas inmediatamente después de terminar sus estudios, cifra que sube al $29.9 \%$ a los 5 años de haberlo hecho. Por otra parte, los hallazgos señalan que las motivaciones tienen efecto positivo sobre la intención emprendedora de los encuestados, siendo la que tuvo mayor valoración "realizar un sueño personal" y las que menos "tener autoridad" y "ser su propio jefe". Además, que el riesgo asociado con la actividad empresarial tiene impacto negativo sobre la creación de empresas. 
Por último, González, Torres y Tinoco (2017) publican los resultados de su investigación en la cual, con una muestra de 618 de estudiantes de licenciatura de la Universidad de Colima en México, encuentran que, mediante modelos de regresión multinomiales, en una situación socioeconómica privilegiada, la experiencia familiar en los negocios, la edad y una alta percepción de espíritu emprendedor y capacidad de trabajo son factores que aumentan la probabilidad de que el estudiante tenga una alta experiencia emprendedora

\subsection{Hipótesis de la investigación}

Teniendo en cuenta el problema planteado, el marco teórico conceptual y los estudios previos señalados, se plantean las siguientes hipótesis de investigación:

Hipótesis 1. Cuanto mayor es la valoración de los factores motivadores para crear empresa en los estudiantes observados, mayor es su actitud emprendedora.

Hipótesis 2. Cuanto mayor es la valoración de las barreras para crear empresa en los estudiantes observados, menor es su actitud emprendedora.

A continuación, se describe la metodología utilizada en este trabajo.

\section{Metodología de la investigación}

\subsection{Descripción de la población, determinación y obtención de la muestra}

Este estudio es de corte transversal, descriptivo y explicativo, ya que busca observar y analizar, para un momento específico, los factores que influyen de manera positiva o negativa sobre la intención emprendedora de los estudiantes objetivo, es decir los 533 matriculados durante el periodo académico de febrero a junio del 2015 en las carreras profesionales de Administración de Empresas o Contaduría Pública de la Universidad del Valle, Sede Norte del Cauca.

Para determinar el tamaño de la muestra, se utilizó la fórmula de Jany (2009) para poblaciones finitas, la cual, con un margen de confianza del 95\% y de error del 5\%, indicó 
que debía ser como mínimo de 223 individuos. El muestreo fue aleatorio y estratificado con asignación proporcional, tomando como variables el programa académico (Administración de Empresas y Contaduría Pública), el género de los alumnos, la jornada (diurna o nocturna), el ciclo académico (fundamentación o inicial y profesional o final) y el estrato socioeconómico (1-3 o 4-6). La recolección de datos se realizó personalmente en junio de 2015, por medio de la aplicación de un cuestionario estructurado, el procesamiento de los datos se realizó utilizando técnicas y herramientas de estadística descriptiva e inferencial, según fuera el objetivo abordado.

La tabla 1 permite ver que la muestra quedó finalmente constituida por 246 estudiantes, de los cuales 73 eran hombres (30\%) y 173 mujeres (70\%); 78 estaban en la jornada diurna (31\%) y 168 en la nocturna (69\%); 118 cursaban el ciclo profesional (48\%) y 128 el de fundamentación (52\%); y, finalmente, 243 eran de estrato socioeconómico 1-3 (99\%) y el solo 3 de los estratos 4-6 (1\%).

Tabla 1. Conformación de la muestra

\begin{tabular}{cccccccccc}
\cline { 3 - 9 } & & \multicolumn{2}{c}{ Género } & \multicolumn{2}{c}{ Jornada } & \multicolumn{2}{c}{ Ciclo } & \multicolumn{2}{c}{ Estrato } \\
\hline Programa & Total & $\mathbf{M}$ & $\mathbf{H}$ & Noc. & Diu. & Prof. & Fund. & $\mathbf{4 - 6}$ & $\mathbf{1 - 3}$ \\
\hline Administración & 140 & 97 & 43 & 85 & 55 & 51 & 89 & 3 & 137 \\
\hline Contaduría & 106 & 76 & 30 & 83 & 23 & 67 & 39 & 0 & 106 \\
\hline Totales & $\mathbf{2 4 6}$ & $\mathbf{1 7 3}$ & $\mathbf{7 3}$ & $\mathbf{1 6 8}$ & $\mathbf{7 8}$ & $\mathbf{1 1 8}$ & $\mathbf{1 2 8}$ & $\mathbf{3}$ & $\mathbf{2 4 3}$ \\
\hline
\end{tabular}

Fuente: elaboración propia.

\subsection{Medición de variables y validación de escalas}

A continuación, se muestran las variables que fueron analizadas y la forma en que se midió cada una, puede verse que en todos los casos se utilizaron escalas tipo Likert.

\subsubsection{Intención emprendedora o de creación de empresa propia}

Para medir esta variable, se les preguntó a los estudiantes cuál era su disposición actual para crear una empresa propia. Se les ofrecieron las siguientes alternativas de respuesta: 1 . Nada dispuesto; 2. Poco dispuesto; 3. Medianamente dispuesto; 4. Dispuesto y 5. Totalmente dispuesto. Es importante señalar que cuando escogían la opción 1 (nada dispuesto), se les pedía no contestar las preguntas sobre motivos para crear la empresa, pero sí las relacionadas con las barreras percibidas. 


\subsubsection{Actitud hacia la creación de empresas}

La actitud hacia la creación de empresas fue medida mediante dos factores: la motivación y las posibles barreras que se pueden encontrar en dicho proceso. La tabla 2 muestra la pregunta que se formuló y las opciones que se utilizaron para conocer los factores que podían motivar la creación de empresas. El valor del indicador Alpha de Cronbach (0.822) cercano a 1 señala que la escala es fiable, es decir, que mide adecuadamente la variable revisada.

Tabla 2. Motivos para la creación de empresas

\begin{tabular}{l}
\hline $\begin{array}{l}\text { Valore el nivel de importancia de cada uno de los siguientes elementos que le motivarían a crear su } \\
\text { propia empresa, teniendo en cuenta la siguiente escala: 1. Nada importante 2. Poco importante 3. Más } \\
\text { O menos importante 4. Importante y 5. Muy importante. }\end{array}$ \\
\begin{tabular}{l} 
Obtener recompensas económicas (Incrementar ingresos, entre otros.) \\
\hline Tener independencia/Autonomía (Libertad personal, ser su propio jefe, entre otros)
\end{tabular} \\
\hline Lograr recompensas personales (Crecimiento personal, probar que es capaz de hacerlo, entre otros) \\
\hline Obtener seguridad familiar y personal (asegurar su futuro y de su familia, tradición familiar, entre otros) \\
\hline Invertir y conseguir un patrimonio personal
\end{tabular}

Fuente: elaboración propia.

Por su parte, la tabla 3 muestra la pregunta que se formuló y las opciones que se consideraron para conocer las barreras o factores que podían desmotivar la creación de empresas, de igual manera que el indicador Alpha de Cronbach tenga un valor de 0.756 (cercano a 1) refleja que la escala es también fiable.

Tabla 3. Barreras percibidas para la creación de empresas

\begin{tabular}{l}
\hline \begin{tabular}{l} 
Valore el nivel de importancia de cada uno de los siguientes elementos que le desmotivarían a crear \\
su propia empresa, teniendo en cuenta la siguiente escala: 1. Nada importante 2. Poco importante 3. \\
Más o menos importante 4. Importante y 5. Muy importante. \\
\hline Riesgo económico (riesgo demasiado elevado, situación económica, falta de un sueldo mínimo ase- \\
gurado, entre otros.) \\
\hline $\begin{array}{l}\text { Tiempo limitado para otras actividades, por tener que trabajar demasiadas horas (tiempo para la } \\
\text { familia, la pareja, entre otros) }\end{array}$ \\
\hline Temor a fracasar \\
\hline Falta de capital para empezar y operar \\
\hline Altos Impuestos
\end{tabular} \\
\hline Desconocimiento sobre las regulaciones para poner en marcha una empresa. \\
\hline Falta de capacitación, asesoría, acompañamiento para iniciar y operar la empresa \\
\hline
\end{tabular}

Fuente: elaboración propia. 


\section{Resultados y discusión}

Enseguida se muestran y discuten los resultados de este trabajo de investigación.

\subsection{Resultados descriptivos}

La tabla 4 muestra el nivel de intención emprendedora de los estudiantes abordados, según las opciones ofrecidas. Se observa que, de su total, el $69.92 \%$ está dispuesto o totalmente dispuesto a crear su propia empresa, el $20.73 \%$ medianamente y solo el 9.35\% tiene ninguna o poca disposición a ello. Así mismo, la intención emprendedora es más alta en la carrera de Contaduría Pública (70.75\%) que en la de Administración de Empresas (69.29\%).

Tabla 4. Nivel de la intención emprendedora

\begin{tabular}{|c|c|c|c|c|c|c|c|c|c|c|c|c|}
\hline & \multicolumn{2}{|c|}{$\begin{array}{c}1 . \\
\text { Nada } \\
\text { dispuesto }\end{array}$} & \multicolumn{2}{|c|}{$\begin{array}{c}2 . \\
\text { Poco } \\
\text { dispuesto }\end{array}$} & \multicolumn{2}{|c|}{$\begin{array}{c}3 . \\
\text { Medianamente } \\
\text { dispuesto }\end{array}$} & \multicolumn{2}{|c|}{$\begin{array}{c}4 . \\
\text { Dispuesto }\end{array}$} & \multicolumn{2}{|c|}{$\begin{array}{c}5 . \\
\text { Totalmente } \\
\text { dispuesto }\end{array}$} & \multicolumn{2}{|c|}{ Totales } \\
\hline & Q & $\%$ & Q & $\%$ & $\mathrm{Q}$ & $\%$ & Q & $\%$ & Q & $\%$ & $\mathrm{Q}$ & $\%$ \\
\hline $\begin{array}{c}\text { En } \\
\text { Administración }\end{array}$ & 4 & 2.86 & 8 & 5.71 & 31 & 22.14 & 54 & 38.57 & 43 & 30.72 & 140 & 57 \\
\hline En Contaduría & 6 & 5.66 & 5 & 4.72 & 20 & 18.87 & 43 & 40.57 & 32 & 30.18 & 106 & 43 \\
\hline En el total & 10 & 4.07 & 13 & 5.28 & 51 & 20.73 & 97 & 39.43 & 75 & 30.49 & 246 & 100 \\
\hline
\end{tabular}

Fuente: elaboración propia.

Los resultado señalados coinciden estrechamente con los de Vargas y Bedoya (2015), en cuanto a que la mayor proporción de la población estudiada estaba realmente interesada en emprender, seguida de la que lo está medianamente y, por último y muy lejos por los que tienen bajo interés en hacerlo o no lo tienen, e igualmente en que la intención emprendedora es más alta en los estudiantes de áreas contables que en de las administrativas.

Por su parte, la tabla 5 muestra que la media de la intención emprendedora en el global de estudiantes encuestados es de 3.87, lo que, al aproximarse al 4.0, indica que en general tienen una buena disposición a crear empresa propia. Estos resultados coinciden con los de otros estudios realizados también en Colombia, como son los de Buelvas et al., 2017 y los de Vargas y Bedoya (2015) en los que, en cada caso, la media está por encima del valor intermedio de las respectivas escalas (de 1 a 5 o de 1 a 7). 
Tabla 5. Media de la intención emprendedora en el global de alumnos

\begin{tabular}{lc}
\hline \multicolumn{2}{c}{ Intención Emprendedora } \\
\hline Media & 3.87 \\
\hline Desviación estándar & 1.04 \\
\hline Mínimo & 1 \\
\hline Máximo & 5 \\
\hline Observaciones & 246 \\
\hline
\end{tabular}

Fuente: elaboración propia.

En la tabla 6 se observa que para el total de estudiantes la calificación promedio de los motivos analizados fue de 4.44, lo que indica que en general tienen una alta motivación para crear empresa propia. Los factores que más los alientan son la seguridad familiar y personal con 4.56 y las recompensas personales con 4.50, y el que menos, las recompensas económicas con 4.28 .

Al contrastar estos resultados con los de Vargas y Bedoya (2015) en estudiantes de nivel tecnológico en Colombia, se vuelve a encontrar, como coincidencia, que el factor motivacional más valorado es la seguridad familiar y personal, pero difieren en que el peor para el presente son las recompensas económicas, mientras que en el estudio de los otros autores fueron las recompensas personales (con 4.05). Es importante, además, hacer notar que en las dos investigaciones las recompensas económicas no están entre los tres primeros factores motivacionales, a pesar de que en ambos casos la mayoría de alumnos son de estratos socioeconómicos modestos.

Tabla 6. Medias de los motivos para la creación de empresa

\begin{tabular}{lc}
\hline \multicolumn{1}{c}{ Motivos } & $\begin{array}{c}\text { Total de } \\
\text { estudiantes }\end{array}$ \\
\hline Recompensas económicas & 4.28 \\
\hline Independencia & 4.45 \\
\hline Recompensas personales & 4.50 \\
\hline Seguridad familiar y personal & 4.56 \\
\hline Invertir & 4.45 \\
\hline Sueño profesional & 4.41 \\
\hline Global motivos & $\mathbf{4 . 4 4}$ \\
\hline
\end{tabular}

Fuente: elaboración propia. 
En la tabla 7 se observa que, para el conglomerado de alumnos observados, la calificación promedio de las barreras analizadas en cuanto a la creación de empresa propia fue de 3.40 , lo que indica que en general las perciben como medianamente importantes, siendo las que más les preocupan la falta de capital con 3.79 y los altos impuestos con 3.43 y las que menos, quedar con tiempo limitado con 3.21 y el temor a fracasar con 3.24.

Tabla 7. Medias de las barreras para la creación de empresa

\begin{tabular}{lc}
\hline \multicolumn{1}{c}{ Barreras } & $\begin{array}{c}\text { Total de } \\
\text { estudiantes }\end{array}$ \\
\hline Riesgo económico & 3.40 \\
\hline Tiempo limitado & 3.21 \\
\hline Temor a fracasar & 3.24 \\
\hline Falta de capital & 3.79 \\
\hline Altos impuestos & 3.43 \\
\hline Desconocimiento regulaciones & 3.35 \\
\hline Falta de capacitación & 3.38 \\
\hline Global barreras & $\mathbf{3 . 4 0}$ \\
\hline
\end{tabular}

Fuente: elaboración propia.

Estos hallazgos coinciden con los de Vargas y Bedoya (2015) en la media para el global de las barreras (3.4), en que los factores que más les preocupan a los dos grupos de estudiantes son, en su orden, la falta de capital y el riesgo económico, y en que los que menos, el temor a fracasar y el quedar con tiempo limitado.

La tabla 8 muestra que, al comparar las medias de los factores motivacionales para los alumnos de Contaduría y Administración en todos los casos, se hayan diferencias pero que solo son estadísticamente significativas las correspondientes a las "recompensas económicas" y la "independencia", en ambos casos superior en los estudiantes de contaduría. Estos resultados podrían inducir a pensar que los estudiantes de contaduría, por la realidad de su entorno laboral en el que es generalmente más difícil encontrar empleo que para los de administración, tienen una mayor tendencia a ser empresarios para lograr su sustento económico e independencia laboral. 
Tabla 8. Medias de los motivos para la creación de empresa propia según carrera

\begin{tabular}{lccc}
\hline \multicolumn{1}{c}{ Motivos } & Administración & Contaduría & $\begin{array}{c}\text { P_valor } \\
\text { Comparación } \\
\text { de medias }\end{array}$ \\
\hline Recompensas económicas & 4.16 & 4.43 & $0.009^{\star \star \star}$ \\
\hline Independencia & 4.36 & 4.58 & $0.032^{\star \star}$ \\
\hline Recompensas personales & 4.48 & 4.52 & 0.772 \\
\hline Seguridad familiar y personal & 4.58 & 4.53 & 0.608 \\
\hline Invertir & 4.40 & 4.53 & 0.224 \\
\hline Sueño profesional & 4.38 & 4.45 & 0.487 \\
\hline Global motivos & $\mathbf{4 . 3 9}$ & $\mathbf{4 . 5 1}$ & $\mathbf{0 . 1 4 3}$ \\
\hline
\end{tabular}

Fuente: elaboración propia.

Por su parte, la tabla 9 indica que, si bien todas las medias de las variables consideradas como barreras tienen calificaciones diferentes según si los estudiantes son de Administración o de Contaduría, solo son estadísticamente significativas las correspondientes a la "falta de capital" y los “altos impuestos", en ambos casos superior en los alumnos de la segunda carrera. Estos resultados podrían llevar a inferir que, por haber una latente saturación en el mercado de los contadores independientes, los estudiantes de esta carrera perciben que puede ser más difícil encontrar capital para crear nuevas empresas en dicha área de negocio, así mismo que al conocer a mayor profundidad el tema de los impuestos, estos les preocupen más.

Tabla 9. Media de las barreras para la creación de empresa según carrera

\begin{tabular}{lccc}
\hline \multicolumn{1}{c}{ Barreras } & Administración & Contaduría & $\begin{array}{c}\text { P_valor } \\
\text { Comparación } \\
\text { de medias }\end{array}$ \\
\hline Riesgo económico & 3.46 & 3.33 & 0.344 \\
\hline Tiempo limitado & 3.22 & 3.19 & 0.819 \\
\hline Temor a fracasar & 3.20 & 3.30 & 0.513 \\
\hline Falta de capital & 3.63 & 4.00 & $0.008^{\star \star \star}$ \\
\hline Altos impuestos & 3.34 & 3.56 & $0.081^{\star}$ \\
\hline Desconocimiento regulaciones & 3.34 & 3.36 & 0.915 \\
\hline Falta de capacitación & 3.31 & 3.47 & 0.265 \\
\hline Global barreras & $\mathbf{3 . 3 6}$ & $\mathbf{3 . 4 6}$ & $\mathbf{0 . 2 4 9}$ \\
\hline
\end{tabular}

Fuente: elaboración propia. 
A continuación, se presentan y discuten los resultados correlacionales o inferenciales.

\subsection{Resultados correlacionales}

Primero, se presentan los modelos conceptuales utilizados para revisar estadísticamente el efecto global o acumulativo de los factores motivacionales (motivación global) y de las barreras (global barreras) sobre la intención emprendedora de los estudiantes observados, y luego los resultados de dichos observaciones.

\subsubsection{Efecto de la motivación global sobre la intención emprendedora}

El siguiente es el modelo de regresión lineal utilizado para validar la hipótesis 1 de este trabajo, es decir que cuanto mayor es la valoración de los factores motivacionales para crear empresa en los estudiantes observados, mayor es su actitud emprendedora:

$$
\begin{gathered}
\text { Intención }=\beta_{0}+\beta_{1} \text { Motivación global } \\
+\epsilon
\end{gathered}
$$

Donde cada factor significa lo siguiente. Intención: disposición actual de crear empresa. $\beta_{0}$ : estimación del promedio de la intención emprendedora. Motivación global: efecto acumulado de los factores considerados como motivacionales. $\beta_{1}$ : estimadores de los parámetros que acompañan las variables y dan razón del aumento o disminución de la intención emprendedora por cada unidad de aumento de ellas. $€$ : Error aleatorio.

La tabla 10 muestra los resultados de la regresión lineal realizada para determinar el efecto de la motivación global sobre la intención emprendedora de los estudiantes encuestados. Es importante ver que el valor de la F (18.401***) señala un excelente ajuste general del modelo definido, que al ser el del vIF 1.0 y la prueba de Durvin Watson estar por debajo de 1.66, se deduce respectivamente que no hay problemas de multicolinealidad ni independencia con los datos utilizados. Así mismo, que el encontrar un $\mathrm{R}^{2}$ del 0.073 indica que la varianza de la intención emprendedora es explicada en un $7.35 \%$ por la motivación global, y que existen entonces otros factores que contribuyen a ella y que pueden ser complementariamente explorados. 
Tabla 10. Efecto de la motivación global sobre la intención emprendedora

\begin{tabular}{lc}
\cline { 2 - 2 } & Total de estudiantes \\
\hline Motivación global & $0.366^{\star * \star}$ \\
$\beta$ & 4.290 \\
$\mathrm{t}$ & $18.401^{\star * \star}$ \\
$\mathrm{F}$ & 1.0 \\
VIF & 0.663 \\
Durvin Watson & 0.073 \\
R2 Ajustado & \\
\hline
\end{tabular}

Fuente: elaboración propia.

La misma tabla muestra que el beta es positivo y significativo en un $99 \%$ de confianza $\left(0.366^{* * * *}\right)$, lo que indica que cada punto de aumento en la motivación global (sumatoria de las recompensas económicas, la independencia, las recompensas personales, la seguridad familiar y personal, conseguir un patrimonio personal y hacer realidad sueños) hace que se incremente en 0.366 puntos en promedio la intención emprendedora de los alumnos. Estos resultados que permiten probar la primera hipótesis del trabajo están en la línea de los hallazgos de Vargas y Bedoya (2015) y los de Yang (2013).

\subsubsection{Efecto del global barreras sobre la intención emprendedora}

El siguiente es el modelo de regresión lineal utilizado para validar las hipótesis 2 de este trabajo, es decir, que cuanto mayor es la valoración de las barreras para crear empresa en los estudiantes observados, menor es su actitud emprendedora.

$$
\begin{gathered}
\text { Intención }=\beta_{0}+\beta_{1} \text { Global barreras } \\
+\epsilon
\end{gathered}
$$

Intención: Disposición actual de crear empresa. $\beta_{0}$ : Estimación del promedio de la intención emprendedora, cuando no hay intervención de las demás variables. Globalbarreras: acumulado del efecto de la valoración de los factores considerados como barreras (riesgo económico, tiempo limitado, temor a fracasar, falta de capital, altos impuestos, desconocimiento de regulaciones y falta de capacitación). $\beta_{1}$ : Estimador del parámetro que acompaña 
la variable y da razón del aumento o disminución de la intención emprendedora por cada unidad de aumento del global barreras. $\epsilon$ : Error aleatorio

La tabla 11 presenta los resultados de la regresión lineal realizada para determinar el efecto del global o acumulado de los factores percibidos como barreras sobre la intención emprendedora de los estudiantes abordados. Se observa que ni la F ni el beta son significativos, además de que el $\mathrm{R}^{2}$ es bastante bajo, por lo que, a pesar de que los vif y el valor del Durbin Watson muestran la no violación de la multicolinealidad e independencia de los datos, en esta investigación no se pudo confirmar la segunda hipótesis planteada, o sea, que no se puede demostrar que el efecto acumulado de la percepción de riesgo económico, tiempo limitado, temor a fracasar, falta de capital, altos impuestos, desconocimiento de regulaciones y la falta de capacitación, desestimula la intención emprendedora de los estudiantes de contaduría y administración. Estos resultados están en contravía con los de Vargas y Bedoya (2015) quienes sí pudieron demostrar en su investigación la misma hipótesis en cuanto a los estudiantes de tecnologías en una ciudad capital.

Tabla 11. Efecto del global barreras sobre la intención emprendedora

Total de estudiantes

\begin{tabular}{lc}
\hline Global barreras & \\
$\beta$ & -0.130 \\
$\mathrm{t}$ & -1.377 \\
$\mathrm{~F}$ & 1.895 \\
VIF & 1.0 \\
DuvinWatson & 0.348 \\
R2 Ajustado & 0.004 \\
\hline
\end{tabular}

Fuente: elaboración propia.

\section{Conclusiones}

Como principales conclusiones de este trabajo desarrollado con 246 estudiantes de las carreras de administración de empresas y de contaduría pública residentes en municipios colombianos pequeños no capitales, y que busca aportar nuevas evidencias empíricas en el campo del emprendimiento, las intenciones emprendedoras y la creación de empresas dentro del ámbito universitario regional, se pueden señalar las siguientes: 
- La zona geográfica objeto de este estudio tiene en sus futuros profesionales un importante potencial empresarial, lo cual se convierte en una ventaja comparativa que debería alentar a sus dirigentes políticos y sociales, así como a los inversionistas a generar un adecuado entorno para el surgimiento de nuevas organizaciones que aprovechen las vocaciones regionales en el marco del proceso de paz colombiano, al cual son sensibles otros países del mundo. Con esto se generarían así nuevos puestos de trabajo que contribuyan a mitigar los problemas sociales de las comunidades correspondientes.

- Contrario a lo frecuentemente preconcebido en cuanto a que obtener mayores ingresos monetarios es el principal factor motivacional para crear empresas, otros elementos tales como la seguridad familiar y las recompensas personales se muestran como los que más impulsan la intención emprendedora de los estudiantes universitarios analizados. En este sentido, los programas públicos y privados de apoyo deben configurar para ellos una oferta que incluya estrategias que tengan en cuenta su arraigo socio-cultural y familiar, sus nuevas aspiraciones de desarrollo personal, pero al mismo tiempo el acceso a las tecnologías que demanda la denominada generación millennial de la cual la mayoría hace parte.

- La percepción de dificultad para conseguir financiamiento y la existencia de una alta tasa impositiva son los factores que más desalientan la creación de nuevas organizaciones empresariales en la población estudiada. Consecuentemente con ello, y al margen de las limitaciones realmente existentes, la academia y entidades como el Sena con su programa "Fondo Emprender" y la Administración de Impuestos de Colombia (DIAN) deberían ocuparse de llevar mayor información sobre las fuentes de financiación y ventajas tributarias existentes en el país para las nuevas empresas.

- De igual manera, el que las medias de algunos de los factores de motivación y de barreras percibidas fueran estadísticamente diferentes para las dos carreras observadas, añade justificaciones para que en los programas de formación en y para el emprendimiento se consideren particularidades, según el campo específico de trabajo de cada profesión. Por último, se debe indicar que, como

\footnotetext{
El Fondo Emprender es un programa gubernamental colombiano del Servicio Nacional de Aprendizaje (sENA) que busca financiar nuevos emprendimientos de aprendices, estudiantes y profesionales. Mayor información en http://www.fondoemprender.com/ SitePages/Home.aspx
} 
era de esperarse, debido a lo ya señalado por la teoría y las investigaciones empíricas precedentes, los factores considerados motivadores mostraron un efecto positivo sobre la intención emprendedora universitaria, por lo cual se debería incrementar su permanente abordaje y estimulo dentro de los programas que buscan fomentarla.

Como limitaciones del trabajo deben señalarse que se abordaron solo dos carreras y ambas de pregrado, pues el haberlo hecho con otros programa académicos, incluyendo posgrados, habría permitido tener una visión más amplia de la intención emprendedora universitaria de la región, en la cual se encuentra la institución en que se hizo el análisis. Entre las futuras acciones de investigación que podrían desprenderse de esta, se pueden mencionar aplicar la encuesta en otras carreras y ciudades pequeñas de otras regiones de Colombia y/u otros países, de tal manera que se aporte evidencia más fuerte acerca de las características del emprendimiento regional; por otra parte, y siguiendo la línea de trabajo de estudios como los de Wibowo, Saptono y Suparno (2018), Oosterbeek, Van Praag y Ijsselstein (2010), Souitaris, Zerbinati y Al-Laham (2007) y Wilson, Kickul y Marlino (2007), podría agregarse al análisis verificar cómo la formación en emprendimiento está influyendo sobre la intención emprendedora de los alumnos de la región estudiada y de las que se consideren posteriormente.

\section{Referencias}

Ajzen, I. (1991). The theory of planned behavior. Organisational Behavior and Human Decision Processes, (50), 179-211.

Ajzen, I. \& Fishbein, M. (1980). Understanding attitudes and predicting social behavior. Englewood Cliffs, NJ: Prentice Hall.

Alonso, P. (2012). La Configuración de la Intención Emprendedora entre académicos responsables de proyectos de investigación en España. Un enfoque de género. (Tesis de doctorado, Universidad de Cantabria, Santander, España).

Audretsch, D. \& Belitski, M. (2007). Entrepreneurship capital and economic growth. Oxford Review of Economic Policy, 23(1), 63-78. 
Bae, T., Qian, S., Miao, C., \& Fiet, J. (2014). The relationship between entrepreneurship education and entrepreneurial intentions. A meta-analytic review. Entrepreneurship theory and practice, 38(2), 217-225.

Buelvas, P., Franco, C., García, G. Gómez, L., López, S., Matíz, F., ...Varela, R. (2017). Actividad Empresarial Colombiana. Reporte GEM Colombia 2016/2017. Cali: Universidad ICESI - Pontificia Universidad Javeriana, Cali - Universidad del Norte - Universidad EAN CECAR - Universidad Cooperativa de Colombia. Recuperado de https://www.researchgate.net/publication/317929591_Actividad_Empresarial_Colombiana_Reporte_GEM_ Colombia_20162017

Chan, M., Escalante M., \& Robles, E. (2015). Comparabilidad en el nivel de emprendimiento de los alumnos de dos universidades: México y Puerto Rico. Revista Internacional Administración y Finanzas, 8(5), 1-14.

Chantson, J. \& Urban, B. (2018). Entrepreneurial intentions of research scientists and engineers. South african journal of industrial engineering, 29(2), 113-126. https://doi. org/10.7166/29-2-1533

Coque, J., Díaz, F., \& López, N. (2013). Factores para la puesta en marcha y el éxito de microempresas asociativas creadas por jóvenes egresados universitarios. Revista de Estudios Cooperativos, (112), 66-94

Dewi, R. \& Martdianty, F. (2012). Student's entrepreneurial intentions by using Theory of Planned Behavior. The Case in Indonesia. The South East Asian Journal, 6(2), 100-143.

Díaz C., Fernández, A., Almodóvar, M., Díaz, Á., \& Fernández, Y. (2017). Intenciones y actividades emprendedoras de los estudiantes de la Universidad de Extremadura: Proyecto GUESS 2013/2014 Universidad de Extremadura. Cáceres España: Editorial Universidad de Extremadura.

Diez, S. (2016) La Actitud Conductual en las Intenciones Emprendedoras. Revista Empresarial, ICE-FEE-UCSG, 1O(2), 42-48.

Fernández, M. (2017). Estudio de las variables que afectan a la intención emprendedora en jóvenes universitarios (Trabajo de grado, Universidad de Sevilla, Sevilla, España).

Galvão, A., Marques, C. S., \& Marques, C. P. (2018). Antecedents of entrepreneurial intentions among students in vocational training programmes. Education and Training, $60(7,8)$, 719-734. https://doi.org/10.1108/ET-03-2017-0034

García, Y., Mendoza, I., \& Romo, L. (2017). Relación entre la Intención de la elección de la carrera de Ingeniería Civil y la iniciativa emprendedora de los estudiantes de una institución de educación superior tecnológica en Hidalgo, México. European Scientific Journal, 13(34), 101-118.

Gómez, S. (2010). Influencia de los factores institucionales sobre la actividad emprendedora corporativa. Un análisis causal (Tesis Doctoral, Universidad de Granada, Granada, España). 
González, D. F. (2004). Incidencia del marco institucional en la capacidad emprendedora de los jóvenes de Andalucía (Tesis doctoral, Universidad de Sevilla, Sevilla, España). Recuperado de http://fondosdigitales.us.es/tesis/tesis/287/incidencia-del-marco-institucional-en-la-capacidad-emprendedora-de-los-jovenes-empresarios-de-andalucia/

González, R., Torres, V., \& Tinoco, M. (2017). Análisis empírico de los determinantes del emprendimiento en estudiantes universitarios. El caso de la Universidad de Colima en México. Economía y Sociedad, 21(36), 43-59.

Gorgievski, M., Stephan, U., Laguna, M., \& Moriano, J. A. (2018). Predicting Entrepreneurial Career Intentions: Values and the Theory of Planned Behavior. Journal of career assessment, 26(3), 457-475. https://doi.org/10.1177/1069072717714541

Goyanes, M. (2015). Apoyo estructural en la intención emprendedora de estudiantes de periodismo y comunicación audiovisual en España. El profesional de la información, 24(1), 55-61.

Guauña, R., A. (2015). Factores psicológicos relacionados con el nivel de intención emprendedora de los estudiantes de administración de empresas y contaduría pública en la Universidad del Valle sede Norte del Cauca (Trabajo de grado, Universidad del Valle, Cali, Colombia).

Jaimes, F., Jamarillo, M., \& Pérez, M. (2017). Factores que inciden en la intención emprendedora de estudiantes del Centro Universitario Tempascaltepec. Revista Venezolana de Gerencia, 22(78), 210-231.

Jany, C. J. (2009). Fundamentos de Muestreo. Investigación Integral de Mercados. Bogotá: McGraw Hill.

Joensuu, S., Varamäki, E., \& Viljamaa, A. (2015). Beyond intentions-what makes a student start a firm? Education+Training, 57(8/9), 853-873.

Kouriloff, M. (2000). Exploring Perceptions of a Priori Barriers to Entrepreneurship: a Multidisciplinary Approach. Entrepreneurship: Theory and Practice, 25(2), 59-79.

Krueger, N. (2000). The cognitive infrastructure of opportunity emergence. Entrepreneurship: Theory \& Practice, 24(3), 5-23.

Krueger, N., Reilly, M., \& Carsrud, A. (2000). Competing models of entrepreneurial intentions. Journal of Business Venturing, 15(5), 411-432. https://doi.org/10.1016/S08839026(98)00033-0

Laguía, A., Moriano, J., Molero, F., \& Gámez, J. (2017). Validación del Cuestionario de Intención Emprendedora en una muestra de estudiantes universitarios de Colombia. Universitas Psychologica, 16(1), 1-14.

Liñán, F. (2004): Intention-based models of entrepreneurship education. Piccolla Impresa / Small Business, (3), 11-35. 
Liñán, F. \& Chen, Y. (2009). Development and Cross-Cultural Application of a Specific Instrument to Measure Entrepreneurial Intentions. Entrepreneurship theory and practice, 33(3), 593-617. https://doi.org/10.1111/j.1540-6520.2009.00318.x

Liñán, F., Moriano, J., \& Jaén, I. (2016). Individualism and entrepreneurship: Does the pattern depend on the social context? International Small Business Journal, 34(6), 760-776.

Marín, L., Rubio, A. Sánchez, M. (2015). El impacto del entorno del estudiante en sus intenciones de crear una empresa cuando finalice sus estudios, Lan Harremanak, 32(1), 504-526.

Mora, P. R. (2011). Estudio de actitudes emprendedoras con profesionales que crearon empresa. Revista EAN, (71), 70-83

Moriano, L. J., Trejo, E., \& Palací, D. F. (2001). El perfil psicosocial del emprendedor un estudio desde la perspectiva de los valores. Revista de Psicología Social, 16(2), 229-243.

Novillo, E., Sarmiento, C., Ollague, J., \& Ramón, D. (2017). Análisis de Actitud Emprendedora en Estudiantes Universitarios: una Perspectiva de Género. INNOVA Research Journal, 2(8), 56-63.

Oosterbeek, H., Van Praag, M., \& Ijsselstein, A. (2010). The impact of entrepreneurship education on entrepreneurship skills and motivation. European economic review, 54(3), 442454. https://doi.org/10.1016/j.euroecorev.2009.08.002

Pallí, C. \& Martínez, L. (2004). Naturaleza y organización de las actitudes. En T. Ibañez, M. Botella, M. Domènech, J. Feliu, L. Martínez, C. Pallí, et al. (Coords.), Introducción a la psicología social (pp. 183-254). Barcelona: EDIUOC.

Park, S. \& Duarte, S. (2015). El perfil del emprendedor y los estudios relacionados a los emprendedores iberoamericanos. Revista Internacional de Investigación en Ciencias Sociales, 11(2), 291-314.

Real Academia Española (RAE) (2017). Definición de 'emprender'. En Diccionario de la lengua española. Recuperado de http://dle.rae.es/?id=Esip2Nv

Rueda, I., Sánchez, L., Blanco, B., \& Fernández, A. (2013). Motivos y frenos al emprendimiento: ¿varía su importancia según el género? Serie estudios del IUDE, 13125. Recuperado de https://drive.google.com/file/d/1abmo-4hvL-ITOEOJ-9qryUGQRrmhFAHV/view

Rueda, S. I., Fernández, L. A., \& Herrero, C. Á. (2012). Estudiantes universitarios Cantabria y emprendimiento: investigaron determinantes psicológicos de la intención de creación de un negocio propio. Faedpyme International Review, 1(2), 9-15.

Sánchez, J. C. Lanero, A., \& Yurrebaso, A. (2005). Variables determinantes de la intención emprendedora en el contexto universitario. Revista de psicología social aplicada, 15(1), 37-59.

Schumpeter, J. S. (1950). Capitalism, Socialism And Democracy. Nueva York: Harper and Row Publisher. 
Shapero, A. (1984). Entrepreneurship in Economic Development. In C. A. Far, (Ed.). Shaping the local Economy. Washintong: International City Management Association.

Shirokova, G., Tsukanova, T., \& Morris, M. (2018). The Moderating Role of National Culture in the Relationship Between University Entrepreneurship Offerings and Student Start Up Activity: An Embeddedness Perspective. Journal of Small Business Management, 56(1), 103-130.

Souitaris, V., Zerbinati, S., \& Al-Laham, A. (2007). Do entrepreneurship programmes raise entrepreneurial intention of science and engineering students? The effect of learning, inspiration and resources. Journal of business venturing, 22(4), 566-591. https://doi. org/10.1016/j.jbusvent.2006.05.002

Timmons, J. A. \& Spinelli, S. (2004) New Venture Creation: Entrepreneurship for the 21st. Century. Boston: McGraw Hill Irwin.

Vainrub, R. (2009). Una guía para Emprendedores: convertir sueños en realidades. México, DF: Pearson Education.

Valencia, A., Cadavid, L., Ríos, D., \& Awad, G. (2012). Factores que inciden en las intenciones emprendedoras de los estudiantes. Revista Venezolana de Gerencia, 17(57), 132-148.

Valencia, A., Montoya, I., \& Montoya, L. (2015). Factores explicativos de las intenciones emprendedoras en estudiantes universitarios. Espacios, 36(5), 1-15.

Valencia, A., Montoya, I., \& Montoya, L. (2016). Intención emprendedora en estudiantes universitarios: Un estudio bibliométrico. Intangible Capital, 12(4), 881-922.

Varela, R. (2008) Innovación Empresarial, Arte y Ciencia en la Creación de Empresas. Bogotá: Pearson Educación.

Vargas, L. \& Bedoya, M. T. (2015). Determinantes psicológicos de la intención de creación de empresas en los aprendices del Centro de Gestión Tecnológica de Servicios del SENA - Cali (Trabajo de grado, Universidad del Valle, Cali, Colombia).

Wibowo, A., Saptono, A., \& Suparno, J. (2018). Does teachers' creativity impact on vocational students' entrepreneurial intention. Journal of Entrepreneurship Education, 21(3), 12-25.

Wilson, F., Kickul, J., \& Marlino, D. (2007). Gender, entrepreneurial self-efficacy, and entrepreneurial career intentions: Implications for entrepreneurship education. Entrepreneurship theory and practice, 31(3), 387-406. https://doi.org/10.1111/j.1540-6520.2007.00179.x

Yang, J. (2013). The theory of planned behavior and prediction of entrepreneurial intention among Chinese Undergraduates. Social Behavior and Personality, 41(3), 367-376. 
Zhao, H., Seibert, S., \& Hills, G. E. (2005). The mediating role of self-efficacy in the development of entrepreneurial intentions. Journal of applied psychology, 90(6), 1265-1272. https://doi.org/10.1037/0021-9010.90.6.1265 